\title{
QUBIC2: A novel biclustering algorithm for large-scale bulk RNA-sequencing and single- cell RNA-sequencing data analysis
}

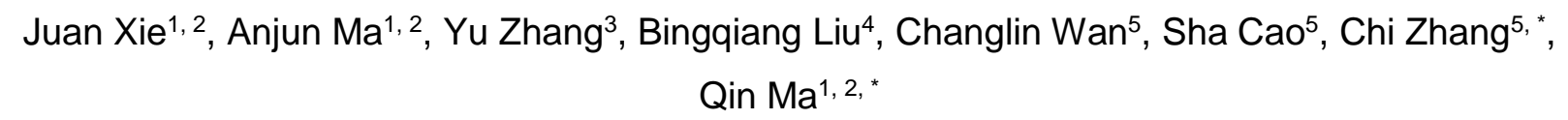

${ }^{1}$ Bioinformatics and Mathematical Biosciences Lab, Department of Agronomy, Horticulture and Plant Science, South Dakota State University, BioSNTR, Brookings, SD, 57007, USA

${ }^{2}$ Department of Mathematics and Statistics, South Dakota State University, Brookings, SD, 57006, USA,

${ }^{3}$ Colleges of Computer Science and Technology, Jilin University, Changchun,130012, China,

${ }^{4}$ School of Mathematics, Shandong University, Jinan, 250100, China, and

${ }^{5}$ Center for Computational Biology and Bioinformatics, Department of Medical and Molecular Genetics, Indiana University, School of Medicine, Indianapolis, IN,46202, USA.

*To whom correspondence should be addressed. Tel: +1 605-688-6315; Email: qin.ma@sdstate.edu. Correspondence is also addressed to Chi Zhang. Tel: +1 317-278-9625; Email: czhang87@iu.edu.

\section{ABSTRACT}

The combination of biclustering and large-scale gene expression data holds a promising potential for inference of the condition specific functional pathways/networks. However, existing biclustering tools do not have satisfied performance on high-resolution RNA-sequencing (RNASeq) data, majorly due to the lack of ( $I$ ) a consideration of high sparsity of RNA-Seq data, e.g., the massive zeros or lowly expressed genes in the data, especially for single-cell RNA-Seq (scRNA-Seq) data, and (ii) an understanding of the underlying transcriptional regulation signals of the observed gene expression values. Here we presented a novel biclustering algorithm namely QUBIC2, for the analysis of large-scale bulk RNA-Seq and scRNA-Seq data. Key novelties of the algorithm include ( $I$ ) used a truncated model to handle the unreliable quantification of genes with low or moderate expression, (ii) adopted the mixture Gaussian distribution and an informationdivergency objective function to capture shared transcriptional regulation signals among a set of genes, (iii) utilized a Core-Dual strategy to identify biclusters and optimize relevant parameters, and (iv) developed a size-based $P$-value framework to evaluate the statistical significances of all the identified biclusters. Our method validation on comprehensive data sets of bulk and single cell RNA-seq data suggests that QUBIC2 had superior performance in functional modules detection and cell type classification compared with the other five widely-used biclustering tools. In addition, 
the applications of temporal and spatial data demonstrated that QUBIC2 can derive meaningful biological information from scRNA-Seq data. The source code for QUBIC2 can be freely accessed at https://github.com/maqin2001/qubic2.

\section{INTRODUCTION}

As next-generation sequencing technologies have become more affordable in these years $(1,2)$, it is possible to generate large-scale biological data with higher resolution, better accuracy, and lower technical variation than the array-based counterparts $(3,4)$. RNA-Seq measures the abundance of RNA transcripts, giving rise to genome-scale gene expression data in a biological sample at a given moment (5). Nowadays, researchers can isolate individual cells from complex organisms and measure transcriptional activity using single-cell sequencing. Hundreds of RNAseq data sets with more than hundreds of sample were emerged in the public domain in the past five years, and their tremendous values have been confirmed in many research areas, e.g., elucidation of cell-type-specific gene regulatory networks (6) and cancer \& complex diseases (79).

The abundance of gene expression datasets provides an opportunity to computationally identify condition based functional gene modules (FGMs), each of which is defined by a similar expression patterns over a certain gene set, which tend to be functionally related or co-regulated by the same transcriptional regulatory signals (TRSs) under a specific condition. Thus, successfully derivation of the FGMs may grant a higher-level interpretation of gene expression data, improve functional annotation of genes, facilitate inference of gene regulatory relationships, and provide a better mechanism level understanding of diseases such as cancer. The identification of FGMs can be naturally modeled as a specific data pattern over unknown subset of genes and samples, and solved with a bi-clustering approach (10), a two-dimensional data mining technique that can simultaneously identify co-expressed genes under a subset of conditions (i.e., samples or cells). This unique feature makes it more useful than clustering when applied to large-scale gene expression data, as genes are usually co-expressed under certain instead of all conditions.

Besides the identification of FGMs in bulk tissue data, a similar formulation may also be applied to scRNA-Seq data, to identify individual cells or cell types as well as their complex interactions under specific stimuli, e.g., cell types classification and clustering. In multicellular organisms,

67 biological function emerges when heterogeneous cell types form complex organs (11). 68 Investigations into organ development, cell function, and disease microenvironment highly 
depend upon an accurate identification and categorization of cell types, sometimes along with their temporal and spatial features (12). Traditionally, a cell type was predicted based on morphological properties or marker proteins, yet this method failed to characterize the full diversity of cells. scRNA-Seq data provides the possibility to group cells based on their genome-wide transcriptome profiles, and several studies have already been carried out using scRNA-Seq data to identify novel cell types, proving its power to unravel the full diversity of cells in human and mouse (13). Mathematically, the problem of scRNA-seq based cell types classification can be naturally formulated as biclustering problems, since the essence is to find sub-populations of cells sharing common expression patterns among subsets of genes.

Substantial efforts have been made in biclustering algorithm and tool development since 2000 (14-26), and a few review studies have provided considerable guidance in choosing suitable algorithms in different contexts (27-29): Eren et al. compared 12 algorithms and concluded that our previously developed method, QUBIC, is one of the top performed methods, as it has achieved the highest performance in synthetic datasets and captured a high proportion of enriched biclusters on real datasets, comparing to Plaid, FABIA, ISA and Bimax, which were also recommended for capturing upregulated biclusters (27). In 2018, Saelens et al. ranked ISA, FABIA and QUBIC as the top biclustering methods in terms of predicting gene modules from human and/or synthetic data (30).

Although numerous bi-clustering methods have been developed for gene expression data analysis, the most existing algorithm are designed and evaluated using microarray rather than RNA-Seq data. One of the unique features of gene expression data derived from RNA-Seq, especially the scRNA-Seq data, is the massive zeros (up to $60 \%$ of all the genes in a cell have read counts being zeros) $(31,32)$. The normalized read counts roughly follow lognormal distributions; however, the raw zero counts of specific genes will lead to negative infinity after logarithmic transformation (33-36), resulting in unquantifiable errors. Therefore, the biclustering methods that are successful for microarray cannot be directly applicable to RNA-Seq data (37), and novel methods taking full consideration of characteristics of RNA-Seq data are urgently needed in the public domain.

In this paper, we developed a novel bi-clustering algorithm, namely QUBIC2, for large-scale RNAseq data analysis. We demonstrated the performance of QUBIC2 on capturing FGMs by applying it to four datasets and benchmarking against five widely used biclustering algorithms. QUBIC2 
103

104

105

106

107

108

109

110

111

112

113

114

115

116

117

118

119

120

121

122

123

124

125

126

127

128

129

130

131

132

133

134

135

136

turned out to be a superior player as it has identified a significant higher proportion of enriched and diverse FGMs. Besides, QUBIC2 can also identify cell types with a higher accuracy, comparing to the five biclustering tools and SC3, a state-of-the-art cell clustering method. Furthermore, we also illustrated the application power of QUBIC2 on inferring time- and spatialrelated insights from two temporal and two spatial scRNA-seq datasets.

\section{RESULTS}

\section{Overall design of QUBIC2}

Inheriting the qualitative representation and graph-theory based model from QUBIC (19), QUBIC2 has four unique features: $(I)$ developed a rigorous truncated model to handle the unquantifiable errors caused by zeros, and used a reliable qualitative representation of gene expression to reflect expression states corresponding to various TRSs; (ii) integrated an information-divergence objective function in the biclustering framework in support of functional gene modules identification; (iii) employed a Core-Dual strategy to optimize consistency level of a to-be-identified bicluster; and (iv) developed a robust $P$-value framework to support statistical evaluation of all the identified biclusters. Details of these four features are showcased as follows (Figure 1).

A mixture of left-truncated Gaussian distributions (LTMG) model was designed to fit the RNA-Seq data, rather than discarding zeros or adding a small constant to original counts $(34,38)$. The basic idea is to treat the large number of observed zeros and low expressions as left censored data in the mixture Gaussian model of each gene $(39,40)$, assuming that the observed frequency of expressions on the left of the censoring point should be equal to the area of the cumulative distribution function of the mixture Gaussian distribution left of the censoring point. Furthermore, we assumed that a gene should receive $K$ possible TRSs under all the conditions, and its expression profile would follow a mixture of $K$ left truncated Gaussian distributions. The LTMG model was applied to fit the expression value of each gene and the gene expression value under a specific condition was labeled to the most likely distribution. Accordingly, a row consisting of discrete values $(1,2, \cdots, K)$ for each gene was generated (Figure $1 \mathrm{~A})$. Then this qualitative row was split into $K$ new rows, such that in the $i^{\text {th }}$ row those labeled initially as $i$ are labeled as 1 , while the rest were labeled as 0 . Finally, a binary representing matrix $M_{R}$ was generated.

A weighted graph $G=(V, E)$ was constructed based on $M_{R}$, where nodes $V$ correspond to genes, edges $E$ connecting every pair of genes (Figure 1B). The edge weight indicates the similarity between the two corresponding genes, which is defined as the number of conditions in which the 
two genes have 1s in $M_{R}$. Intuitively, two genes from a bicluster should have a heavy edge in $G$ innately while two random genes may have a heavy edge only accidentally. Hence, a bicluster should correspond to a maximal subgraph of $G$, with edges typically heavier than the edges of an arbitrary subgraph. Identifying all the biclusters equals to identifying all the heavy subgraphs in $G$, which is an NP-hard problem. Therefore, a heuristic strategy was designed as follows.

The algorithm would iterate a seed list $(S)$, which is the sorted list of edges in $G$ in the decreasing order of their weights (i.e., $\left.w\left(e_{1}\right) \geq w\left(e_{2}\right) \geq \cdots, w\left(e_{|E|}\right)\right)$. An edge $e_{i j}=g_{i} g_{j}$ is selected as a seed if and only if at least one of $g_{i}$ and $g_{j}$ is not in any previously identified biclusters, or $g_{i}$ and $g_{j}$ are in two nonintersecting biclusters in terms of genes. QUBIC2 first built a core bicluster from a seed and then expanded to recruit more genes and conditions into a to-be-identified bicluster, until the Kullback-Leibler divergence score (KL score) was locally optimized. It was proposed based on the assumption that the difference between a bicluster and its background should be larger than the difference between an arbitrary same-size submatrix and its background. The KL score of a bicluster was designed to quantify this difference as the larger of the difference was, the larger of the score is (Figure 1C. See Methods for details).

During bicluster expansion, the algorithm controlled the consistency level for a bicluster, which is defined as the minimum ratio of the number of $1 \mathrm{~s}$ in a column/row and the number of rows/columns in the bicluster. In QUBIC, a pre-specified value c $(0<c \leq 1.0)$ was used to control the overall consistency level of the bicluster. While this parameter was dynamically optimized by a Dual searching method in QUBIC2 (Figure 1D-E), giving rise to a submatrix $\left(I, \mathcal{J}\right.$ of $M_{R}$ (i.e., a bicluster) with optimized consistency level and maximal KL score can be identified. Biclusters expanded using Dual strategy tend to be more significant than those without Dual (See Example S1 in Supplementary File 1).

Furthermore, for the first time, a statistical framework based on the size of the biclusters was implemented to calculate a $P$-value for each of the identified biclusters. The problem of assessing the significance of identified biclusters was formulated as calculating the probability of finding at least one submatrix enriched by 1 from a binary matrix with given size, with a beta distribution employed during the process. This $P$-value framework enables users systematically evaluate the statistical significance of all the identified biclusters, especially for those from less-annotated organisms (Figure 1F). 


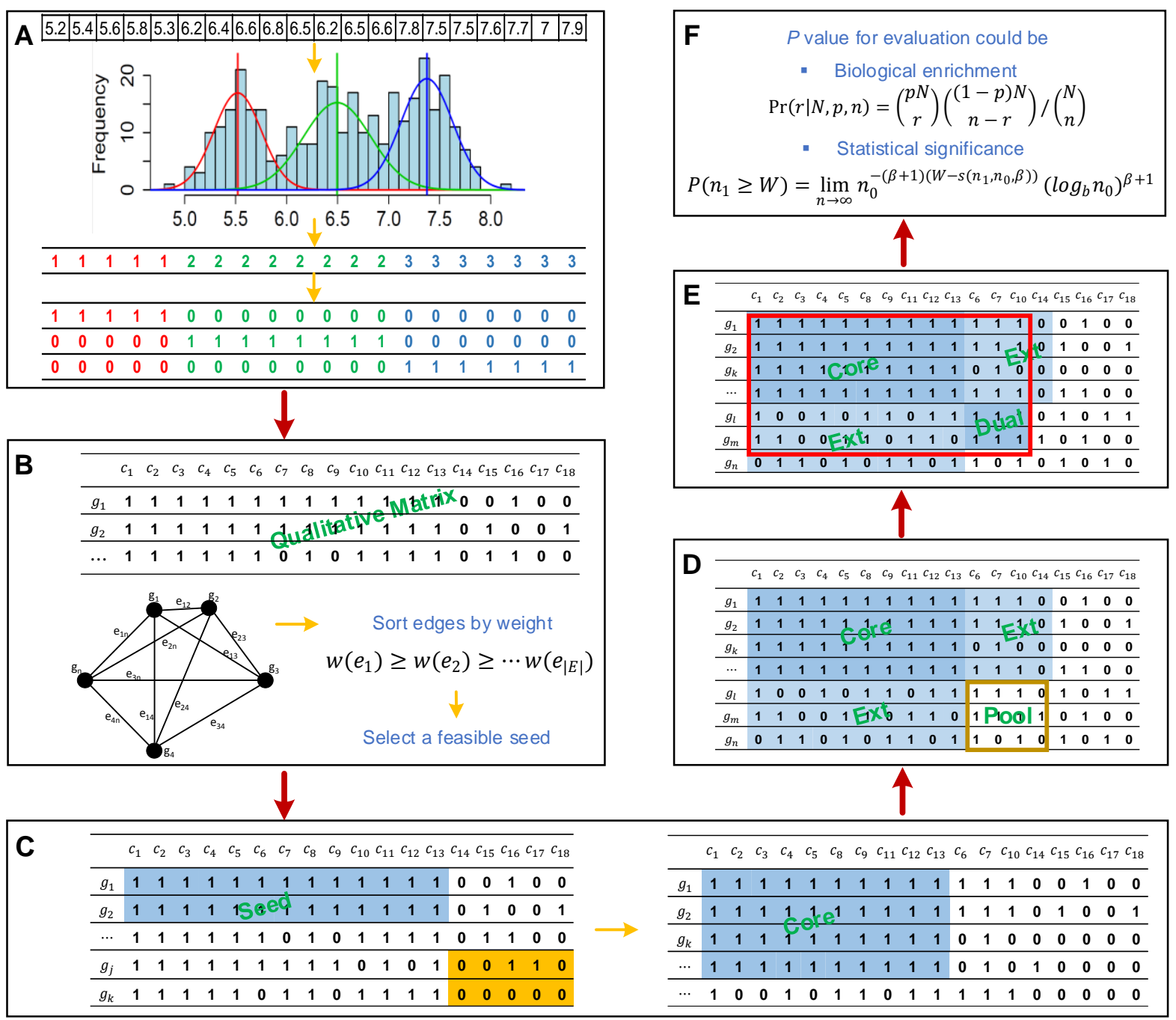

Figure 1. QUBIC2 workflow. A. Discretization of gene expression data from RNA-Seq. The LTMG model

173 will be applied to fit each gene's expression profile. A representing row for each gene will be generated with

174 integers denote the most likely component distribution that each value belongs to. Then this representing row will be split into multiple rows. Finally, a binary representing matrix will be generated; B. Graph construction and seed selection. A weighted group will be constructed based on the representing matrix

177 from A. By sorting the edges in decreasing order of their weight, and an initial seed list will be obtained.

178 QUBIC2 will select a feasible seed from the list; C. Build an initial core based on the selected seed. During seed expand, QUBIC2 will search for genes with higher weight with the seed. In case of two genes have

180 the same weight, the one with higher $\mathrm{KL}$ score will be selected. Thus, gene $\mathrm{k}(\mathrm{KL}=0.1914)$ instead of gene $181 \mathrm{j}(\mathrm{KL}=0.0622)$ will be added to the core first; $\mathbf{D}$. Expand core and determine pool. QUBIC2 will expand the 182 core vertically and horizontally to recruit more genes and conditions under a preset consistency level, respectively. The intersected zone created by extended genes and conditions as a Dual searching pool (brown box); E. Dual search in the pool and output the bicluster with genes and conditions that come from 
185

186

187

188

189

190

191

192

193

194

195

196

197

198

199

200

201

202

203

204

205

206

207

208

209

210

211

212

213

214

215

216

217

218

Core and Dual as final bicluster (red box); F. Statistical evaluation of identified biclusters based on either biological annotations or the size of the bicluster.

\section{Functional gene modules detection from RNA-Seq data}

Compared with five biclustering algorithms (Bimax(18), ISA(41), FABIA(20), Plaid(15), and QUBIC(19), with more details in Table S1 of Supplementary File 1), the performance of QUBIC2 in identifying FGMs was systematically evaluated using four gene expression datasets: a simulated RNA-Seq dataset based on an in-house method (22,846 rows $\times 100$ columns), a bulk RNA-Seq dataset from Escherichia coli (E. coli, 4,497 rows $\times 155$ columns), a bulk RNA-Seq dataset from TCGA (3,084 rows $\times 8,555$ columns), and a scRNA-Seq dataset from human embryos $(3,798$ genes $\times 90$ cells). For the identified biclusters from a specific tool, precision showcases the fraction of biclusters whose genes are significantly enriched with certain biological pathways (i.e., relevance), and recall reflects the fraction of captured known modules/pathways among all known modules in a functional annotation database, e.g., KEGG (42) and RegulonDB (43) (i.e., diversity). The harmonic mean value of precision and recall, referred to as the $F$ score, was used as the integrated criteria in performance evaluation.

Evaluation studies usually used default parameters of the to-be-analyzed tools, which were optimized for specific benchmark datasets. However, when applied to datasets coming from a different organism (e.g., E. coli vs. human), or be acquired by other technologies (e.g., microarray vs. RNA-Seq), the default parameters often fail to achieve satisfying performance and need further optimization/adjustment. To minimize the biases in performance comparison among multiple tools, for each of the four datasets, we run the six tools under more than 50 parameter combinations by adjusting their critical parameters around default/recommended values (see Methods and Table S2 in Supplementary File 1). Then the $F$ score of identified biclusters under each parameter combination was calculated. In this way, we can test a tool's robustness and infer how sensitive of its performance is to parameter adjustment, besides the basic performance comparison among different tools.

As showcased in Figure 2, QUBIC2 achieved the highest median $F$ scores and the highest $F$ scores with the default parameter on all the four datasets, and its $F$ scores were significantly higher than the second-best algorithms in all the comparison circumstances (Wilcoxon test $P$ value $<0.01$ ). QUBIC2 performed well in both precision and recall, indicating that the identified FGMs are relevant and diverse; and it had relatively small variance, while the performance of 
some algorithms on certain dataset was very sensitive to parameter change (e.g., FABIA on $E$. coli). Regarding median $F$ scores, QUBIC was the second-best algorithm on simulated data, $E$. coli RNA-Seq data, and human scRNA-Seq data, while FABIA was the second-best one for TCGA

222 data. As regards the default settings, QUBIC ranked as the top ones on simulated data and $E$.

223 coli data, and ISA and Plaid had relative higher rank on TCGA data. ISA was generally very stable,

224 and its variances were the smallest on three datasets. As for Bimax, although its recall was

225 relatively low, it was characterized with high precision on the four datasets. It is noteworthy that

226 QUBIC2 is the only program, among all the six biclustering algorithms, which did not encounter a

227 dramatic performance drop on scRNA-Seq data compared to RNA-Seq data, suggesting the

228 unique applicative power of QUBIC2 on FGMs detection from scRNA-Seq data (Figure S1 in

229 Supplementary File1).

230

231 Furthermore, the performance of all the biclustering algorithms on $E$. coli data was better than on

232 human data, with the possible reason that $E$. coli data has more completed functional annotation

233 and affects the evaluation of module significance. Therefore, for less annotated organisms, we need a statistical evaluation framework for all the identified biclusters.

235

A
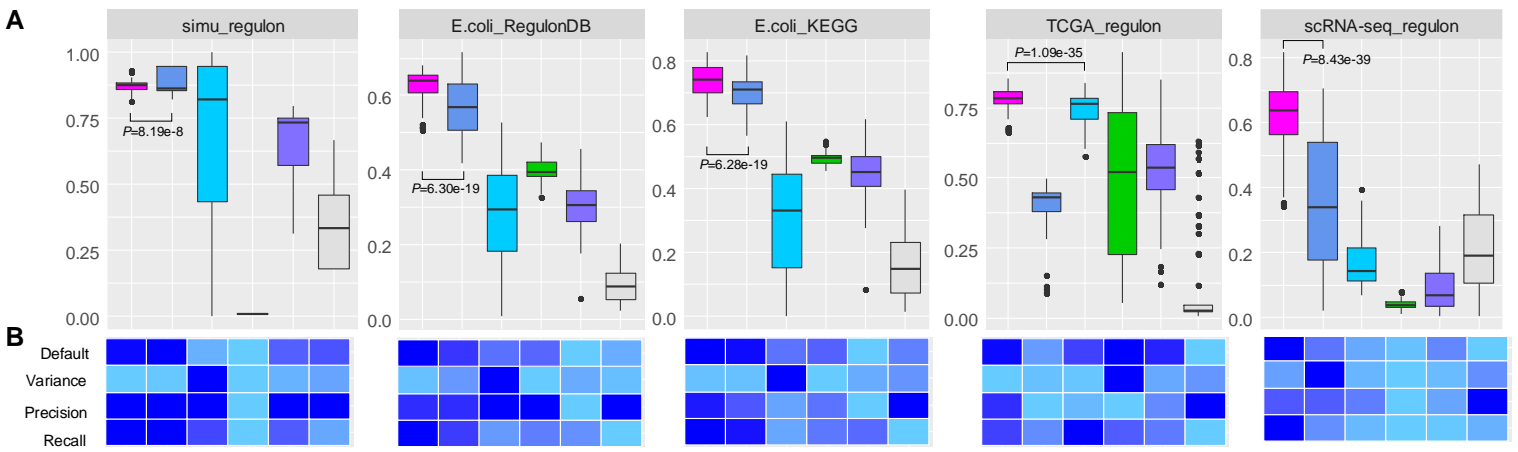

METH QUBIC2 官 QUBIC FABIA

Plaid

官 Bimax

Figure 2. Overall performance comparison between QUBIC2 and five popular biclustering methods based on the agreement between identified biclusters and known modules. A. Distribution of $F$ scores on each of the four datasets under multiple runs $(n>40)$. Black line in the box denote median value, whiskers denote $10 \%$ and $90 \%$ percentiles, while the box denotes $25 \%$ and $75 \%$ percentiles; $B$. relative performance of six algorithms in terms of $F$ score under default parameters, variance of $F$ scores under multiple sets of parameters, median value for the precision and median value for the recall, respectively (normalized over six algorithms). Note that the variance of $F$ scores depends on the increment of parameters, and therefore only indicative. 


\section{A statistical evaluation framework for identified biclusters}

248 The significances of gene modules from the identified biclusters were usually evaluated by 249 pathway enrichment analysis. However, many organisms (including human) have limited

250 functional annotations supported by experimentally verifications, which makes a systematic

251 evaluation of all identified biclusters non-trivial. To fill this gap, a statistical method was proposed

252 in this study, which can calculate a $P$-value for a bicluster purely based on their size (number of

253 genes and conditions).

254

255 Interestingly, we found that there is a strong association between the $P$-values of biclusters 256 calculated via pathway enrichment analysis (named knowledge-based $P$-value) and the 257 corresponding size-based $P$-values. Specifically, spearmen correlation tests were conducted 258 between size-based $P$-values and five groups of knowledge-based $P$-value (see Methods). The 259 average spearman correlation coefficients $(\rho)$ were higher than 0.40 (ComTF_ $\rho=0.48, T F \_\rho=0.56$, 260 KEGG_ $\rho=0.42$, SEED_ $\rho=0.43$ and ECO_ $\rho=0.42$ ), and the average $p$-values for the correlation 261 test were smaller than 0.01. As showcased in Figure 3A, all the $\rho$ s in the five groups are positive. 262 In addition, $\rho$ s related with regulatory pathways (i.e., $\mathrm{TF}_{-} \rho$ and ComTF_ $\rho$ ) were generally larger 263 than $\rho$ s those related to metabolic pathways (i.e., KEGG_ $\rho$ and SEED_ $\rho$ ). This indicated that the 264 size-based $P$-value seemed to be more suitable for the evaluation of biclusters' regulatory 265 significance. Furthermore, all the corresponding $p$-values were less than 0.05 (Figure 3B), 266 suggesting that the correlations between knowledge-based $P$-values and size-based $P$-values 267 were statistically significant at the 0.05 level. In addition, the parameter $f$ which controls the level 268 of overlaps between biclusters had a negative association with $\rho$ (Figure S2 in Supplementary 269 File1), suggesting that the size-based $P$-values would have a stronger association with 270 knowledge-based $P$-values when the overlaps between biclusters are relatively low.

271
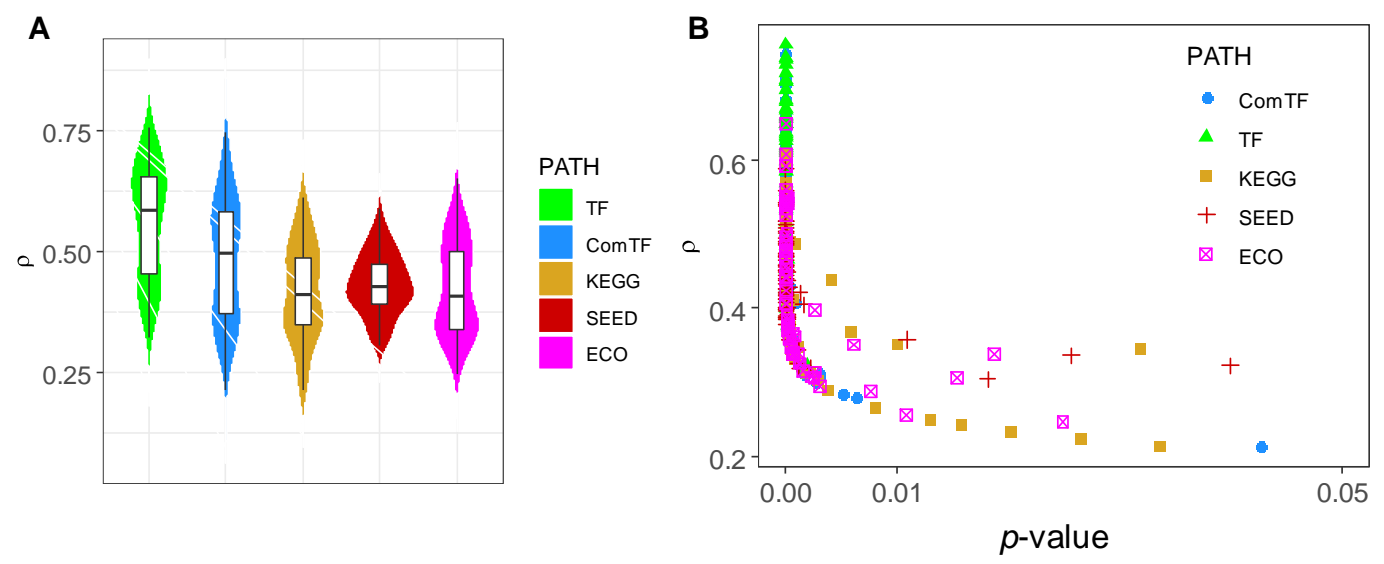
273 Figure 3. A. The distribution of correlation coefficients $(\rho)$ between $P$-value obtained from enrichment

274 analysis and size-based $P$-value. We run QUBIC2 under 63 different parameter settings, and $\rho$ was

275 calculated under each run; $\boldsymbol{B}$. Scatter plot of $\rho$ and $p$-value. The $y$-axis denotes $\rho$, the correlation coefficient

276 for the spearman association test, the $x$-axis denotes the $p$-value of the association test. Note that to

277 distinguish, italic lowercase $p$ was used to denote the $p$-value of the Spearman correlation test, while italic

278 uppercase $\mathrm{P}$ was used to denote the significance of biclusters.

279

\section{Cell type classification based on ScRNA-Seq data}

281 The above sections demonstrated the outstanding performance of QUBIC2 on FGMs

282 identification and its unique feature of statistical evaluation for all the identified biclusters. In this

283 section, we showed the predictive power of biclustering methods on cell types identification from

284 scRNA-Seq data.

285

286 We developed a pipeline to group cells into different types with the assumption that two cells belonging to the same bicluster have a higher likelihood of being the same cell type than two randomly selected cells (see Methods). Briefly, a biclustering tool was first used to identify biclusters from a scRNA-Seq expression data. Then, a weighted graph $G=(C, E)$ was constructed to model the relationship between cell pairs, where nodes $C$ represent cells, edges $E$ connect pairs of cells, and edge weight indicates the number of biclusters that the two corresponding cells appear in simultaneously. Finally, cell types were predicted via the Markov Cluster Algorithm (MCL) clustering on the weighted graph (Figure 4A).

For each of the six biclustering methods in Figure 2, we applied this pipeline to a benchmark dataset with 20,214 genes and 90 cells (41), which have been experimentally classified into seven types (46). The Adjusted Rand Index (ARI) was adopted as the evaluation criteria to access the agreement between predicted cell types and these 'ground truth' (46). Two more external validation criteria, namely Jaccard Index (JI) and Fowlkes Mallows Index (FW), were also used here aiming to provide a comprehensive evaluation.

As Figure 4B showed, QUBIC2 and QUBIC were the top two biclustering tools, respectively, in terms of median values on the three criteria. Both surpassed the performance of SC3 (41), which was used as the benchmark (median value from 100 runs) and was denoted by the red dash line

305 in each panel of Figure 4B. In addition, ISA always demonstrated the smallest variance across 306 the three validation criteria. The FW values of each tool were more stable than other two values. 
A

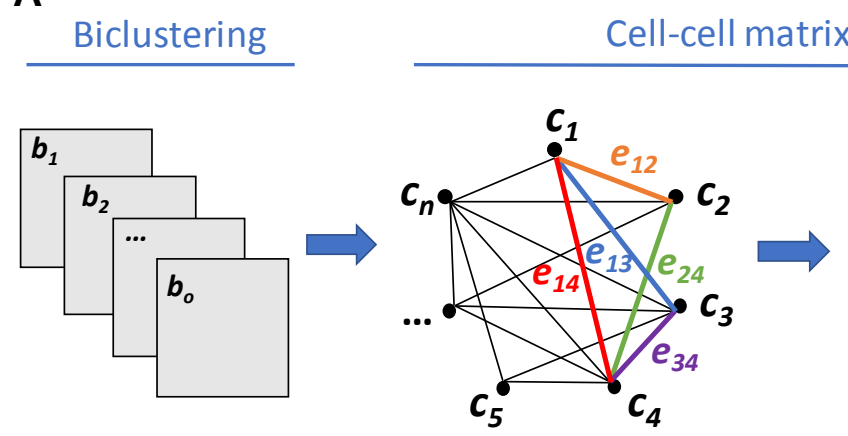

B
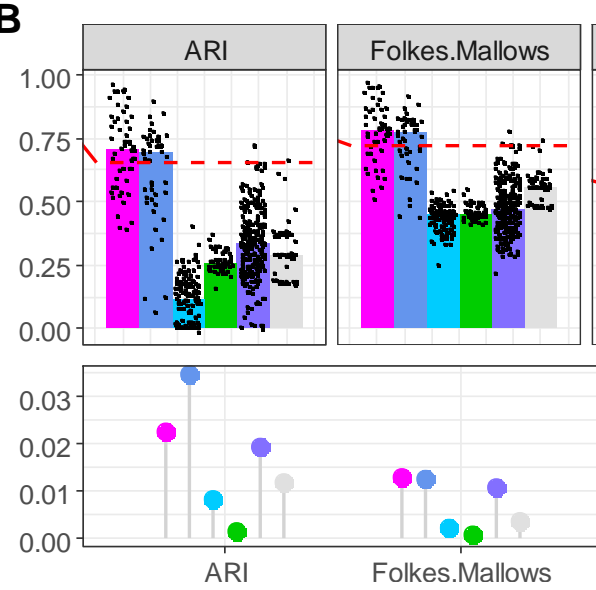

Jaccard

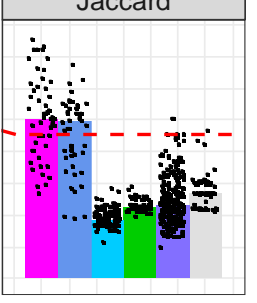

312

313

314

315

316

317

318

319

320

321

322

323

324

325

Figure 4. A. Computational pipeline for cell type classification. This pipeline consists of three steps: biclustering, generation of weighted cell-cell matrix and clustering using MCL. The input is biclusters and output is cell type labels; B. Benchmark of QUBIC2 against five popular biclustering algorithms. Upper layer: each panel shows the similarity between the inferred labels and the reference labels quantified by the four indices, i.e., Adjusted Rand Index (ARI), Folkes Mallows's index and Jaccard Index, respectively. Each algorithm was applied $>40$ times to the same dataset to evaluate accuracy and stability. The three indices were calculated for each run of the respective methods (black dots). Bars represent the median of the distribution of black dots. The red dash lines correspond to the benchmark performance of SC3 (ARI: 0.6549 , FW: 0.7243 , Jl: 0.5671 ). Lower layer: the variance of each tool in terms of three validation criteria; C. Sankey diagram comparing the 7 clusters obtained with SC3 (left layer) and 6 clusters obtained with QUBIC2 (right layer). The middle layer corresponds to the seven reference clusters. The widths of the lines linking nodes from two layers correspond to the number of cells they have in common. 


\section{QUBIC2 inferred the temporal and spatial organization of cells from scRNA-Seq data}

327 When spatial and temporal information is available, scRNA-Seq can reveal more biological insights beyond cell types. In this section, QUBIC2 was applied on two temporal (and) and two spatial scRNA-Seq datasets, respectively, to explore the temporal and spatial organization of cells.

Five biclusters were identified by QUBIC2 from a time series lung scRNA-Seq data (GSE52583), which consists of 152 cells collected at E14, E16 and E18, respectively (47). Three of the five biclusters contain time-specific cells. In particular, bicluster BC002 consists of cells exclusively from E14; bicluster BC003 contains cells that only from E16; and bicluster BC004 has cells coming from E18 (Figure 5A). Functional enrichment analyses of the component genes from these three biclusters were carried out based on DAVID (48) and the results showed that genes in BC002 mainly related to cell cycle, cell division, and mitosis; BC003 genes were enriched with ribosome, translation, and structural constituent of ribosome; and spliceosome-related genes were grouped in BC004 (see details in Supplementary File 2).

In addition to identifying biclusters corresponding to specific time point, QUBIC2 can also be used data with mouse dendritic cells (DCs) collected at $1 \mathrm{~h}, 2 \mathrm{~h}, 4 \mathrm{~h}$ and $6 \mathrm{~h}$ after treatment with pathogenic agent lipopolysaccharide (LPS) and untreated controls (GSE48968) (49). In total, 51 biclusters were identified in the datasets treated with LPS. For each bicluster, the Fisher exact test was conducted on its constituting samples to assess if significant over-representation by any time points could be found within the bicluster. For those biclusters showing significant association with the time-course, a pathway enrichment analysis was conducted to infer the biological characteristics of the bicluster. In the end, 30 biclusters that are significantly over-represented by one or several consecutive time points were identified in the LPS dataset $(\alpha=0.005, P<1 e-22)$, and six of them showed distinct time dependence (Figure 5B). Specifically, bicluster BC013 consists of untreated samples and samples collected at $1 \mathrm{~h}$, which represents the earliest response to LPS and enriches multiple immune response pathways. Bicluster BC005 consists largely of untreated samples and samples collected at $1 \mathrm{~h}$ and $2 \mathrm{~h}$, which also is enriched with immune response pathways but with more responses to a virus, $\mathrm{T}$ cell chemotaxis and so on.

$356 \mathrm{BC009}$ and BC001 are enriched by samples collected at $1 \mathrm{~h}$ and $2 \mathrm{~h}$, covering a wider range of 357 stress-response pathways, suggesting that the activation of stress response pathways and 358 altered metabolisms as secondary responses after the early immune response. BC025 and 359 BC002 consist of samples collected at $4 \mathrm{~h}$ and $6 \mathrm{~h}$, and their genes enrich pathways associated 
with alterations in cell morphogenesis, migration, cell-cell junction and so on. Overall these observations suggest that our analysis can identify all the major responses to the LPS treatment in a time-dependent manner. Detailed pathways enriched by the six biclusters are given in Figure S3 in Supplementary File 1. The detailed information of these biclusters is given in Supplementary File 3.

A

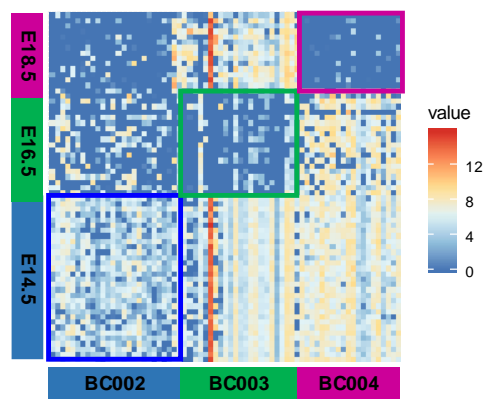

B

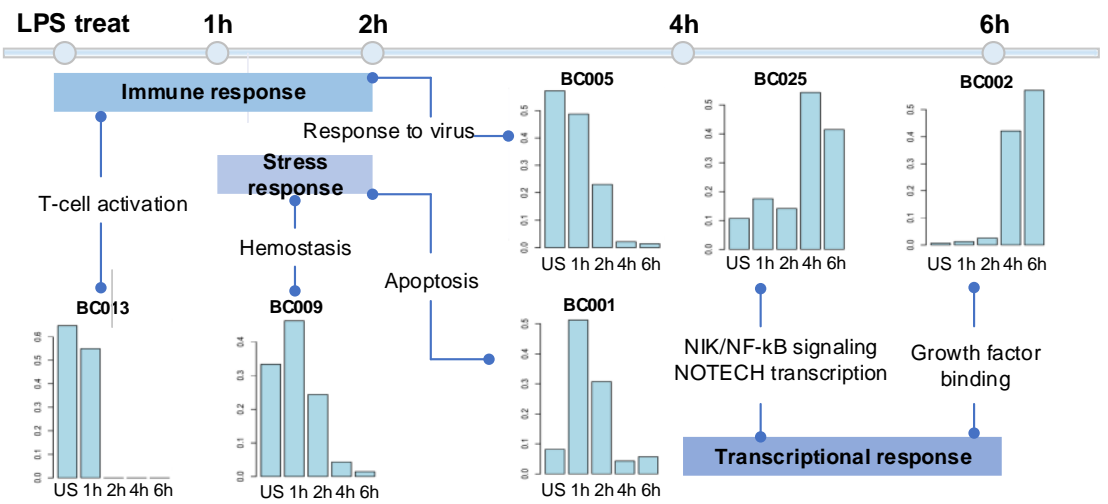

Figure 5. A. Visualization of three biclusters (BC002, BC003, and BC004) selected based on the specificity to time point; B. Time-dependent distribution of cells in six selected biclusters identified in the LPS data. In each histogram, the five bars from left to right show the proportion of the untreated samples and samples collected at $1 \mathrm{~h}, 2 \mathrm{~h}, 4 \mathrm{~h}$ and $6 \mathrm{~h}$ after the LPS treatment.

Then QUBIC2 was applied to a mouse spatial scRNA-Seq dataset with 280 cells. The cells were classified into five clusters that correspond to five well-defined morphological layers in (50) (Figure 6A). Five biclusters were predicted. Among them, the bicluster BC000 consists of cells mainly from the granular layer; the bicluster BC001 contains cells from the mitral layer and glomerular layer; and the bicluster BC002 contains cells mainly from the olfactory nerve layer (Figure 6B). Functional annotation showed that BC000 mainly enriches plasma membrane, cell membrane, and cell projection; BC001 enriches synapse, neuron projection, and cell projection; and BC002 enriches cell projection (Details in Supplementary File 4).

Finally, another spatial scRNA-Seq dataset (GSE60402) with samples dissected from three mouse medial ganglionic eminence tissues and known spatial coordinates was analyzed. QUBIC2 was applied and 37, 40, and 120 biclusters were identified in the mutant, wild-type 1, and wild-type 2 datasets, respectively (Details in Supplementary File 5). Further investigation on the spatial distribution of cells in each bicluster showed that all the four spatial biclusters with distinct expression patterns by cell cycle, cell morphogenesis, and neuron development genes, as reported in the original study (51), were identified by QUBIC2 . It is noteworthy that the outliers 
with highly expressed stem cell markers tend to be located at the intermediate region between two adjacent (or overlapping) biclusters in the three datasets as shown in Figure 6D and 6F. Our interpretation is that these location-dependent expression patterns may be caused by parallel and independent differentiations from common stem cells.

A

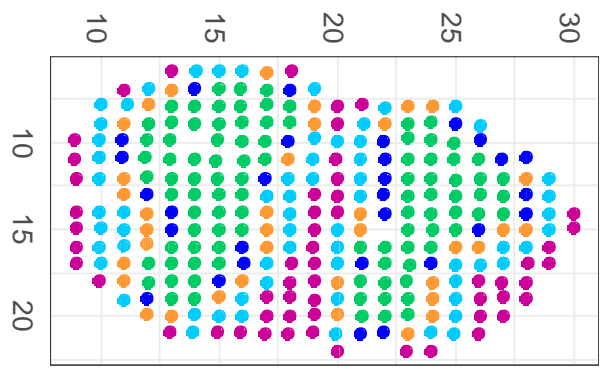

B

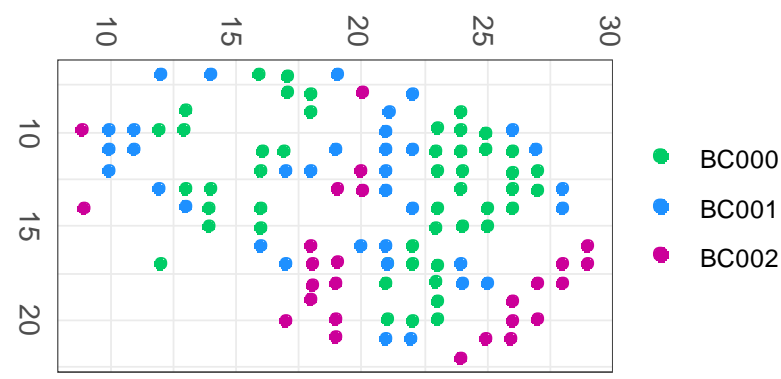

C

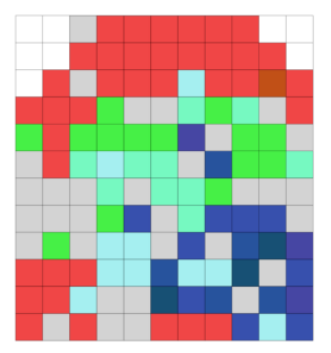

D

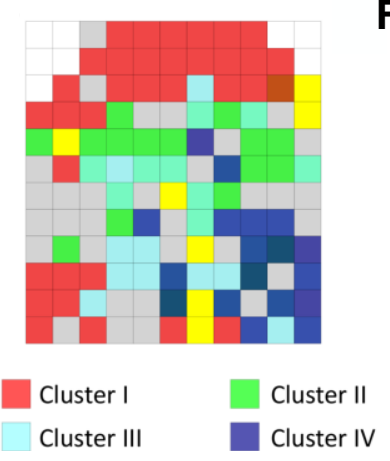

E

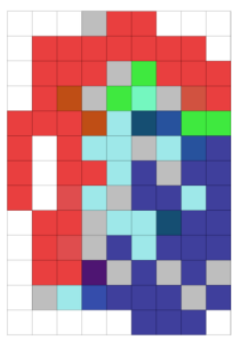

F

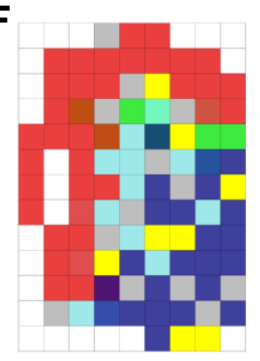

Outlier cells with stemness
391

392

393

394

395

396

397

398

399

400

401

402

403

404

405

406

407

Figure 6. A. The coordinates of cells correspond to five morphological layers (1. Granular cell layer; 2. Mitral cell layer; 3. Outer plexiform layer; 4. Glomerular layer; 5. Olfactory nerve layer); B. The coordinates of cells from three selected biclusters; $\mathbf{C}$. The spatial coordinates of samples in the four biclusters identified in wild-type 1 mouse; Colors red, green, cyan and dark blue represent samples in four different biclusters; D. In addition to the coordinates of bicluster samples, the yellow cubes represent significant outlier samples; E. The same information as in $C$ except the samples are from wild-type 2 mouse; $\mathbf{F}$. The same information as in $\mathrm{D}$ except the samples are from wild-type 2 mouse.

\section{METHODS AND MATERIALS}

\section{Data acquisition}

A total of four expression datasets were used in the Functional gene modules detection from RNA-Seq data section, that is, one synthetic RNA-Seq data, one E. coli RNA-Seq data and two human datasets (one RNA-Seq and one scRNA-Seq). The synthetic dataset was simulated using our in-house simulation method (details in Simulation of co-regulated gene expression data section). It contains 22,846 genes and 100 samples. A total of 10 co-regulated modules was embedded in this dataset, covering 2,240 up-regulated genes. The E. coli RNA-Seq data 
consists of 4,497 genes and 155 samples, which was integrated and aggregated by our group. In short, 155 fastq files were downloaded from ftp://ftp.sra.ebi.ac.uk/vol1/fastq/ using the sratoolkit (v2.8.1, https://github.com/ncbi/sra-tools/wiki/Downloads), and they are processed following quality check (FastQC), reads trimming (Btrim), reads mapping (HISAT2) and transcript counting (HTseq). Then, raw read counts were RPKM normalized. The human RNASeq data contains 3,084 genes and 8,555 samples, which was obtained from (30). The scRNASeq data was downloaded from (13) as an RPKM expression matrix with 20,214 gene and 90 cells and then 3,798 genes were kept for the analysis in this study by removing the genes without annotation.

Multiple sets of known modules/biological pathways were provided or collected to support the enrichment analysis of the above four datasets. For synthetic data, the 10 groups of pre-defined up-regulated genes were used as co-regulated modules. For E. coli data, we used five kinds of biological pathways, which are complex regulons and regulons extracted from the RegulonDB database (version 9.4, accessed on 05/08/2017), KEGG pathways collected from the KEGG database (accessed on 08/08/2017), SEED subsystems from the SEED genomic database (accessed on 08/08/2017) (44), and EcoCyc pathways from the EcoCyc database (version 21.1, as of 08/08/2017) (45). Complex regulons were defined as a group of genes that are regulated by the same transcription factor (TF) or the same set of TFs. In total, 457 complex regulons, 204 regulons, $123 \mathrm{KEGG}$ pathways, 316 SEED subsystems, and 424 EcoCyc pathways were retrieved, respectively. For the human TCGA and scRNA-Seq data, we used three sets of modules provided by (30).

One golden-standard scRNA-Seq data (52) was downloaded from https://scrnaseq-publicdatasets.s3.amazonaws.com/manual-data/yan/nsmb.2660-S2.csv in the cell type classification section. It consists of 20,214 genes and 90 cells, where the cells were assigned into seven subgroups with the true cell subtypes information provided in (52).

The time series lung scRNA-Seq dataset (GSE52583) with 152 cells and 15,174 genes from was downloaded from (47). The cells were collected at three time points: E14, E16, and E18. Another time series scRNA-Seq data with 527 cells and 13991 genes (GSE48968) was downloaded from the GEO database, in which the RPKM values are available.

The Mouse olfactory bulb spatial transcriptomic data was downloaded from (50), which contains 280 cells and 15,981 genes. Ståhl et al. (50) classified the cells into five clusters that correspond 
442 to well-defined morphological layers. The cells use coordinates as IDs, and the cell layers 443 information was manually extracted using the ST viewer

444 (https://github.com/SpatialTranscriptomicsResearch/st viewer), based on the coordinate

445 information (see Supplementary File 6). The raw reads of mouse spatial scRNA-Seq data

446 GSE60402 was retrieved from the SRA database $(53,54)$, and the RPKM values for it were

447 calculated using software packages TopHat (55) and Cufflink (56). GSE60402 was split into three

448 subsets according to sample information. The detailed information of the selected and split

449 datasets is listed in Table 1.

450

451

Table 1. Summary of GSE60402

\begin{tabular}{lcccc}
\hline $\begin{array}{c}\text { GEO } \\
\text { Accession ID }\end{array}$ & Data ID & Description & \#Cells & \#Genes \\
\hline GSE60402 & GSE60402-Mutant & From Gfra1 mutant sample & 94 & 11094 \\
GSE60402 & GSE60402-Wildtype1 & From wild type mouse 1 & 124 & 10037 \\
GSE60402 & GSE60402-Wildtype2 & From wild type mouse 2 & 94 & 10714
\end{tabular}

454 To accurately model the gene expression profile of RNA-Seq and scRNA-Seq data, we 455 specifically developed a mixed Gaussian model with left truncation assumption. Denotes the log 456 transformed FPKM, RPKM or CPM expression values of gene $\mathrm{X}$ over $N$ conditions as $\mathrm{X}=$ $457\left\{x_{1}, \ldots x_{n}\right\}$, we assumed that $x_{j} \in X$ follows a mixture of $k$ Gaussian distributions, corresponding to $458 k$ possible TRSs. The density function of $x_{j}$ is:

$$
p\left(x_{j} ; \Theta\right)=\sum_{i=1}^{k} \alpha_{i} p\left(x_{j} ; \theta_{i}\right)=\sum_{i=1}^{k} \alpha_{i} \frac{1}{\sqrt{2 \pi} \sigma_{i}} e^{\frac{-\left(x_{j}-\mu_{i}\right)^{2}}{2 \sigma_{i}^{2}}}
$$

And the density function of $X$ is:

462 where $\alpha_{i}$ is the mixing weight, $\mu_{i}$ and $\sigma_{i}$ are the mean and standard deviation of $i^{\text {th }}$ Gaussian 463 distribution, which can be estimated by an EM algorithm with given X:

$$
\Theta^{*}=\underset{\Theta}{\arg \max } L(\Theta ; X)
$$


Parameters $\Theta$ can be estimated by iteratively running the estimation (E) and maximization (M) steps. In this study, $Z_{c u t}$ is set for each gene as the logarithm of the minimal non-zero $\mathrm{RPKM} / \mathrm{FPKM} / \mathrm{TPM}$ value in the gene's expression profile. The EM algorithm is conducted for $K=$ $1, \ldots, 9$ to fit the expression profile of each gene and the $K$ that gives the best fit is selected according to the Bayesian Information Criterion $(\mathrm{BIC})$ :

$$
B I C=-2 \ln \left(\Theta^{*}\right)+3 K \ln (N)
$$

where $K$ is the number of TRS, $K$ is the number of conditions. $K$ that minimizes the BIC will be selected.

Then the original gene expression values will be labeled to the most likely distribution under each cell. In detail, the probability that $x_{j}$ belongs to distribution $i$ is formulated by:

$$
p\left(x_{j} \in T R S i \mid K, \Theta^{*}\right) \propto \frac{\alpha_{i}}{\sqrt{2 \pi \sigma_{j}^{2}}} e^{\frac{-\left(x_{j}-\mu_{i}\right)^{2}}{2 \sigma_{i}^{2}}}
$$

And $x_{j}$ is labeled by TRS $i$ if $p\left(x_{j} \in T R S i \mid K, \Theta^{*}\right)=\max _{i=1, \cdots, K}\left(p\left(x_{j} \in T R S i \mid K, \Theta^{*}\right)\right)$. In such a way, a row consisting of discrete values $(1,2, \ldots, K)$ for each gene will be generated.

\section{KL score}

A Kullback-Leibler divergence score (KL score) is introduced in QUBIC 2 to guide candidateselection and biclustering optimization. The $\mathrm{KL}$ score of a bicluster is defined as:

$$
K L_{B}=\frac{1}{N} \sum_{j=1}^{N} \sum_{i \in\{0,1\}} R(i, j) \times \log \frac{R(i, j)}{Q(i, j)}+\frac{1}{M} \sum_{k=1}^{M} \sum_{i \in\{0,1\}} C(i, k) \times \log \frac{C(i, k)}{P(i, k)}
$$

where $N$ and $M$ are the numbers of rows and columns of a submatrix $B$ in $M_{R}$, respectively. $R(i, j)$ represents the proportion of element $i$ in row $j$ of $B, Q(i, j)$ is the proportion of $i$ in the corresponding entire row, $C(i, k)$ is the proportion of $i$ in column $k$ of $B$, and $P(i, k)$ is the proportion of $i$ in the entire corresponding column.

Meanwhile, the KL score for a gene quantify the similarity between a candidate gene $j$ and a bicluster, which is defined as follows:

$$
K L_{j}=\sum_{i \in\{0,1\}} R(i, j) \times \log \frac{R(i, j)}{Q(i, j)}
$$

where $R(i, j)$ represent the proportion of $i$ under corresponding columns of the current bicluster. 


\section{Simulation of co-regulated gene expression data}

496 We utilized a single cell RNA-Seq dataset of human melanoma (58) (with 22,846 genes and 4,645

497 cells) to simulate bulk tissue RNA-Seq data with known co-regulated modules. Specifically, a

498 single cell RNA-Seq pool consists counts data of 4,466 cells of six annotated cell types namely

499 B-, T-, endothelial, fibroblast, macrophage and cancer cells were constructed. The top 1,000 cell

500 type specifically expressed genes of each cell type were identified by using $Z$ score of the mean

501 of each gene's expression level in each cell type.

For each round of simulation, the number of to be simulated bulk tissue samples and co-regulation modules is first defined. Then the genes of each co-regulation module denoted as $X_{k}$, will be specified by randomly selecting $M_{k}$ genes from the top 1,000 cell type specifically expressed genes of one cell type. A co-regulation strength matrix $P$ is then simulated from a bimodal distribution over $(0,1)$, with $P[i, k]$ denotes the proportion of cells with the transcriptional regulatory signal of co-regulation module $k$ in bulk sample $i$. A bulk tissue data is simulated by randomly drawing cells from the cell pool by following a multinomial distribution, with predefined parameters and the total number of cells. For co-regulation module $k$ in bulk sample $i$, genes $X_{k}$ in a proportion $P[i, k]$ of the selected cells of the cell type corresponds to $k$ are perturbed by an $\mathrm{X}$-fold increase of the gene expression. Then the bulk data $i$ with simulated co-regulations are formed by summing the perturbed gene expression profile the selected cells and normalized to RPKM expression scale. The Pseudo code of the simulation approach is provided Method $\mathrm{S} 1$ in Supplementary File 1.

The rationales of this simulation approach include (1) gene expression level and noise in the bulk data are purely simulated by sum of real single-cell data, without using artificially assigned expressions scale and noise; (2) co-regulation genes are modeled as a specific fold increase of a number of cell-type-specific genes in a particular subset of the cells, which characterizes the heterogeneity of transcriptional regulation among cells in a tissue; (3) multiple co-regulation modules in specific to different cell types can be simultaneously simulated. Hence, we believe the gene expression data simulated by this way can satisfactorily reflect genes co-regulated by a perturbed transcriptional regulation signal in real bulk tissue data.

\section{Evaluation of the functional modules}

527 The capability of algorithms to recapitulate known functional modules are assessed using 528 precision and recall. First, for each identified bicluster, we use the $P$-value of its most enriched 
529 functional class (biological pathway) as the $P$-value of the bicluster. Specifically, the probability of 530 having $x$ genes of the same functional class in a bicluster of size $n$ from a genome with a total of $531 N$ genes can be computed using the following hypergeometric function(59):

$$
\mathrm{P}(X=x \mid N, p, n)=\frac{\left(\begin{array}{c}
p N \\
x
\end{array}\right)\left(\begin{array}{c}
1-p) N \\
n-x
\end{array}\right)}{\left(\begin{array}{c}
N \\
n
\end{array}\right)}
$$

where $p$ is the percentage of that pathway among all pathways in the whole genome. The

$$
P-\text { value }=\mathrm{P}(\mathrm{X} \geq \mathrm{x})=1-\mathrm{P}(\mathrm{X}<\mathrm{x})=1-\sum_{i=0}^{x-1} \frac{\left(\begin{array}{c}
p N \\
i
\end{array}\right)\left(\begin{array}{c}
(1-p) N \\
n-i
\end{array}\right)}{\left(\begin{array}{l}
N \\
n
\end{array}\right)}
$$

The bicluster is deemed enriched with that function if its $p$-value is smaller than a specific cutoff (e.g., 0.05).

Given a group of biclusters identified by a tool under a parameter combination, the precision is defined as the fraction of observed biclusters significantly enriched with the one biological pathway/known modules (Benjamini-Hochberg adjusted $p<0.05$ ),

For recall, we compute the fraction of known modules that were rediscovered by the algorithms,

\section{7}

Finally, the harmonic mean of precision and recall were calculated to represent the performance of an algorithm on a given dataset and parameter setting, denoted as $F$ score:

$$
\text { Recall }=\frac{\# \text { of significant modules }}{\# \text { of modules }}
$$

$$
F=\frac{2}{\frac{1}{\text { Precision }}+\frac{1}{\text { Recall }}}
$$

551 To make sure the evaluation is as fair as possible, for each dataset, we select the first 30 552 biclusters.

\section{Parameter adjustment of biclustering tools}

555 To assess the robustness of selected algorithms' performance, each tool is run multiple times by 556 varying parameters that affect the size and number of biclusters. In general, parameters are 557 adjusted around their default or recommended (if available) value. The parameters that varied are 
560

561

\begin{tabular}{ccc}
\hline Algorithm & Implementation & Parameters \\
\hline Bimax & R package 'biclust' & minr, minc, number \\
ISA & R package 'isa2' & set.seed \\
FABIA & R package 'fabia' & alpha, spl, spz, cyc, $\mathrm{p}$ \\
Plaid & R package 'biclust' & row.release, col.release, max.layer \\
QUBIC & R package 'QUBIC' & $\mathrm{f}, \mathrm{c}, \mathrm{k}, \mathrm{o}$ \\
QUBIC2 & $\mathrm{C}++$ & $\mathrm{f}, \mathrm{c}, \mathrm{k}, \mathrm{o}$
\end{tabular}

listed in Table2, and details about the range and increment of parameters can be found in Supplementary File.

Table 2. Main parameters adjusted for each algorithm
562

563

564

565

566

567

568

569

570

571

572

573

574

575

576

577

578

\section{Spearman correlation test}

QUBIC2 was run on the E. coli RNA-Seq data in Figure 2 under 63 parameter settings. For each setting, around 100 biclusters were identified. Five sets regulatory or metabolic pathways were extracted from four databases of E. coli (RegulonDB, KEGG, SEED (46) and EcoCyc (47)) to support this association study. In specific, for each set of $\sim 100$ biclusters obtained under the same settings, six groups of $P$-values for all these biclusters were calculated, with five knowledgebased groups and one size-based group. Spearman correlation test was conducted to investigate the rank-order correlation among the six groups of $P$-values. Five correlation coefficients $(\rho)$, which demonstrated the extent of correlation between size-based $P$-values and five biological knowledge-based $P$-values, as well as five corresponding $p$-values, were recorded from the test. Note that the $p$-value of correlation test denotes the probability of observing such a correlation or even stronger correlation, under the null hypothesis that no correlation exists. For simplicity, the correlation coefficient between the size-based $P$-value and biological knowledge-based $P$-value was prefixed with the name of a pathway, e.g., TF_ $\rho$ and KEGG_ $\rho$. In the end, a total of $5 \times 63$ $\rho$ (63 parameter settings, each with five $\rho$ ) ) and a same number of $p$-values were obtained. 


\section{Cell type classification pipeline}

By using biclustering, we can group genes and cells simultaneously. However, since biclustering aims to find sets of genes that are co-expressed across a subset of conditions, it is possible that genes may co-expressed across multiple cell types. Therefore, one bicluster may consist of cells from different types, and cells from the same types may appear in different biclusters. In a word, it is not guaranteed that one bicluster corresponds to one cell type. However, it is assumed that two cells from a bicluster are more likely to be of the same subtypes than the two cells that are randomly selected. It is believed that biclusters can capture this feature to some extent. If there are multiple biclusters and when we condense them together, we can distinguish sets of cells belonging to the same type from sets of cells that are grouped by chance.

Based on the above idea, we developed a pipeline to obtain cell type classification based on biclustering results (Figure 4A). First, a biclustering tool was applied to the expression data (rows represent genes and columns represent cells) to identify a set of biclusters. Then a weighted graph $G=(C, E)$ was constructed to model the relationship between cell pairs among biclusters. A node $c_{i}$ in $G$ represented a cell, and $e_{i, j}$ represented the edge connecting $c_{i}$ and $c_{j}$, where $i \neq j$. We assigned weight $w_{i, j}$ to $e_{i, j}$ to represent the number of biclusters that contain both $c_{i}$ and $c_{j}$. Intuitively, a higher $w_{i, j}$ value indicates that $c_{i}$ and $c_{j}$ are simultaneously involved in more biclusters, hence, are more likely to be the same cell type than cell pairs with lower weight. A symmetrical cell-cell matrix with diagonal as 0 was then constructed to record $w_{i, j}$ and Markov Cluster Algorithm (MCL) was performed to cluster cells into cell types and produce cell labels. In specific, the MCL clustering was run 100 times by varying inflation factor, resulting 100 cell labels. A binary similarity matrix was constructed for each cell label: if two cells belong to the same cluster, their similarity is 1 ; otherwise, the similarity is 0 . Then a consensus matrix was built by averaging all similarity matrices. The resulting consensus matrix was clustered using hierarchical clustering with complete agglomeration, and the clusters were inferred at the k level of the hierarchy.

\section{External cluster validity indices}

External validation measures the extent to which cluster labels match externally supplied class labels. Generally, they are based on counting the pairs of points on which two classifiers agree/disagree. Denote two partitions of the same data set as $\mathrm{R}$ and $\mathrm{Q}$. The reference partition, $R$, encode the class labels, i.e., it partitions the data into k known classes. Partition $Q$, in turn, partitions the data into $v$ categories, which is the one to be evaluated. 
Adjusted Rand Index (ARI) is defined as

$$
A R I=\frac{a-\frac{(a+c)(a+b)}{d}}{\frac{(a+c)+(a+b)}{2}-\frac{(a+c)(a+b)}{d}}
$$

615 a: Number of pairs of data objects belonging to the same class in $\mathrm{R}$ and the same cluster in $\mathrm{Q}$.

$616 b$ : Number of pairs of data objects belonging to the same class in $\mathrm{R}$ and different clusters in $\mathrm{Q}$.

617 C: Number of pairs of data objects belonging to different classes in $\mathrm{R}$ and the same cluster in $\mathrm{Q}$.

$618 d$ : Number of pairs of data objects belonging to different classes in $\mathrm{R}$ and different clusters in $\mathrm{Q}$.

619 Terms $a$ and $d$ are measures of consistent classifications (agreements), whereas terms $b$ 620 and $\mathrm{c}$ are measures of inconsistent classifications (disagreements).

622 Jaccard Index is defined as:

$$
J I=\frac{a}{a+b+c}
$$

624 The Jaccard Index can be seen as a proportion of good pairs with respect to the sum of nonneutral (good plus bad) pairs.

Folkes-Mallow's index is defined as

Fowlkes-Mallow's index can be seen as a non-linear modification of the Jaccard coefficient that also keeps normality.

\section{Pathway enrichment analysis}

633 Pathway enrichment analysis is conducted and the statistical significance of each enriched 634 pathway is assessed by using a hypergeometric test (statistical significance cutoff $=0.005$ ) 635 against 4,725 curated gene sets in the MsigDB database, which includes 1,330 canonical KEGG, 636 Biocarta and Reactome pathways, and 3,395 gene sets representing expression signatures 637 derived from experiments with genetic and chemical perturbations, together with 6,215 Mouse 638 GO terms each containing at least 5 genes $(62,63)$.

\section{CONCLUSION}

641 QUBIC2 is a novel biclustering algorithm developed for bulk RNA-Seq and scRNA-Seq data 642 analysis in this study. It has four unique characteristics: $(I)$ used a left-truncated mixture model to 643 fit the log-transformed RPKM/CPM/TPM values of each gene and qualitatively represent gene 
644

645

646

647

648

649

650

651

652

653

654

655

656

657

658

659

660

661

662

663

664

665

666

667

668

669

670

671

672

673

674

675

676

expression; (ii) integrates an information-divergence objective function in the biclustering framework; (iii) applies a Dual strategy to optimize consistency level of a to-be-identified bicluster; and (iv) develops a robust $P$-value framework to evaluate the significance of all the identified biclusters. QUBIC2 proved to have significant advantages in the functional module detection area, outperforming five widely-used biclustering methods based upon our test on four datasets. The proposed $P$-value calculation method based on bicluster size did make sense, which may facilitate the evaluation of all the identified biclusters, especially from less-annotated organisms. The cell type classification pipeline, based on QUBIC2, worked well and outperformed the state-of-the-art performance of SC3. By utilizing time-dependent data, QUBIC2 discovered biclusters specific to time point and identified a cascade of immune responses to the external pathogenic treatment. From the spatial transcriptomic data, QUBIC2 discovered that spatially adjacent single cells may have high co-expression patterns, and particularly, two distinct spatially clustered cells may be derived initially from the same stem cell. We believe that QUBIC2 can serve biologists as a useful tool to extract novel biological insights from large-scale RNA-Seq data (The tutorial for QUBIC2 program is provided in Supplementary File 7).

\section{DISCUSSION}

Single-cell sequencing has enabled new transcriptome-based studies, including the study of distinct responses by different cell types in the same population when encountered by the same stimuli or stresses, and identification of the complex relationships among different cells in complex biological environments such as tissues. However, to fully excavate the potential of scRNA-Seq data, we must overcome several technical challenges.

As sequencing costs decrease, larger scRNA-Seq datasets will become increasingly common; thus, the scalability to large dataset and efficiency of tools will become more and more important. Currently, the discretization and Dual searching functions of QUBIC2 are time consuming on large-scale datasets. Based on our test, it takes 17 minutes to discretize a dataset with 4,297 rows and 466 columns (a desktop with 48.0GB memory, Intel Core i7-6700 and 3.40GHz). Given a dataset with 22,846 genes and 100 conditions, the running time while using Dual strategy are generally 2 minutes longer than that without Dual. The openMP method will be implemented in the EM steps for discretization and more efficient heuristics algorithm will be designed to optimize the dual searching of biclustering. 
702

703

704

705

706

707

708

709

710

711

712

713

714

715

716

717

Another challenge involves the interpretation of time-series and spatial data. For example, in the GSE52583 data, QUBIC2 could only separate cells collected at different time points, yet the further differentiation stage information was not captured. For the mouse olfactory bulb data, QUBIC2 did not separate cells from adjacent layers. To deal with this drawback, we need to combine biclustering with other statistical methods specifically designed for time series and spatial gene expression data.

It is noteworthy that many other kinds of methods can be used for gene expression data analysis. Forty-two module detection tools covering five main approaches were reviewed in (30) and the authors concluded that decomposition methods outperformed all other strategies, including biclustering methods. Meanwhile, they also observed that QUBIC and FABIA had higher performance on human and synthetic data. We compared two top rated decomposition methods and two top clustering methods with QUBIC2 and QUBIC on a human scRNA-Seq data; and the results showed that QUBIC2 surpassed both decomposition and clustering methods (Figure S4 in Supplementary File 1). In the future, we will carry out more comprehensive comparison between QUBIC2 and other decomposition and network-based methods, aiming to give a systematical evaluation of the power of computational techniques on scRNA-seq data.

\section{REFERENCES}

1. Van Dijk, E.L., Auger, H., Jaszczyszyn, Y. and Thermes, C. (2014) Ten years of nextgeneration sequencing technology. Trends in genetics, 30, 418-426.

2. Goodwin, S., McPherson, J.D. and McCombie, W.R. (2016) Coming of age: ten years of next-generation sequencing technologies. Nature Reviews Genetics, 17, 333-351.

3. Marioni, J.C., Mason, C.E., Mane, S.M., Stephens, M. and Gilad, Y. (2008) RNA-seq: an assessment of technical reproducibility and comparison with gene expression arrays. Genome research, 18, 1509-1517.

4. Nagalakshmi, U., Wang, Z., Waern, K., Shou, C., Raha, D., Gerstein, M. and Snyder, M. (2008) The transcriptional landscape of the yeast genome defined by RNA sequencing. Science, 320, 1344-1349.

5. Wang, Z., Gerstein, M. and Snyder, M. (2009) RNA-Seq: a revolutionary tool for transcriptomics. Nature reviews genetics, 10, 57-63.

6. Aibar, S., Gonzalez-Blas, C.B., Moerman, T., Huynh-Thu, V.A., Imrichova, H., Hulselmans, G., Rambow, F., Marine, J.C., Geurts, P., Aerts, J. et al. (2017) SCENIC: single-cell regulatory network inference and clustering. Nat Methods, 14, 1083-1086.

7. Prince, M.E., Sivanandan, R., Kaczorowski, A., Wolf, G.T., Kaplan, M.J., Dalerba, P., Weissman, I.L., Clarke, M.F. and Ailles, L.E. (2007) Identification of a subpopulation of cells with cancer stem cell properties in head and neck squamous cell carcinoma. Proc Natl Acad Sci U S A, 104, 973-978.

8. Navin, N., Kendall, J., Troge, J., Andrews, P., Rodgers, L., McIndoo, J., Cook, K., Stepansky, A., Levy, D., Esposito, D. et al. (2011) Tumour evolution inferred by singlecell sequencing. Nature, 472, 90-94. 
9. Xu, X., Hou, Y., Yin, X., Bao, L., Tang, A., Song, L., Li, F., Tsang, S., Wu, K., Wu, H. et al. (2012) Single-cell exome sequencing reveals single-nucleotide mutation characteristics of a kidney tumor. Cell, 148, 886-895.

10. Ulitsky, I., Maron-Katz, A., Shavit, S., Sagir, D., Linhart, C., Elkon, R., Tanay, A., Sharan, R., Shiloh, Y. and Shamir, R. (2010) Expander: from expression microarrays to networks and functions. Nat Protoc, 5, 303-322.

11. Jaitin, D.A., Kenigsberg, E., Keren-Shaul, H., Elefant, N., Paul, F., Zaretsky, I., Mildner, A., Cohen, N., Jung, S., Tanay, A. et al. (2014) Massively Parallel Single-Cell RNA-Seq for Marker-Free Decomposition of Tissues into Cell Types. Science, 343, 776-779.

12. Shekhar, K., Lapan, S.W., Whitney, I.E., Tran, N.M., Macosko, E.Z., Kowalczyk, M., Adiconis, X., Levin, J.Z., Nemesh, J., Goldman, M. et al. (2016) Comprehensive Classification of Retinal Bipolar Neurons by Single-Cell Transcriptomics. Cell, 166, 13081323.e1330.

13. Kiselev, V.Y., Kirschner, K., Schaub, M.T., Andrews, T., Yiu, A., Chandra, T., Natarajan, K.N., Reik, W., Barahona, M., Green, A.R. et al. (2017) SC3: consensus clustering of single-cell RNA-seq data. Nat Methods, 14, 483-486.

14. Kluger, Y., Basri, R., Chang, J.T. and Gerstein, M. (2003) Spectral biclustering of microarray data: coclustering genes and conditions. Genome research, 13, 703-716.

15. Lazzeroni, L. and Owen, A. (2002) Plaid models for gene expression data. Statistica sinica, 61-86.

16. Madeira, S.C. and Oliveira, A.L. (2009) A polynomial time biclustering algorithm for finding approximate expression patterns in gene expression time series. Algorithms Mol Biol, 4, 8.

17. Bergmann, S., Ihmels, J. and Barkai, N. (2003) Iterative signature algorithm for the analysis of large-scale gene expression data. Physical review E, 67, 031902.

18. Prelić, A., Bleuler, S., Zimmermann, P., Wille, A., Bühlmann, P., Gruissem, W., Hennig, L., Thiele, L. and Zitzler, E. (2006) A systematic comparison and evaluation of biclustering methods for gene expression data. Bioinformatics, 22, 1122-1129.

19. Li, G., Ma, Q., Tang, H., Paterson, A.H. and Xu, Y. (2009) QUBIC: a qualitative biclustering algorithm for analyses of gene expression data. Nucleic Acids Res, 37, e101.

20. Hochreiter, S., Bodenhofer, U., Heusel, M., Mayr, A., Mitterecker, A., Kasim, A., Khamiakova, T., Van Sanden, S., Lin, D., Talloen, W. et al. (2010) FABIA: factor analysis for bicluster acquisition. Bioinformatics, 26, 1520-1527.

21. Barkow, S., Bleuler, S., Prelić, A., Zimmermann, P. and Zitzler, E. (2006) BicAT: a biclustering analysis toolbox. Bioinformatics, 22, 1282-1283.

22. Cheng, K.O., Law, N.F., Siu, W.C. and Lau, T.H. (2007) BiVisu: software tool for bicluster detection and visualization. Bioinformatics, 23, 2342-2344.

23. Wu, C.J. and Kasif, S. (2005) GEMS: a web server for biclustering analysis of expression data. Nucleic Acids Res, 33, W596-599.

24. Zhou, F., Ma, Q., Li, G. and Xu, Y. (2012) QServer: a biclustering server for prediction and assessment of co-expressed gene clusters. PloS one, 7, e32660.

25. Kaiser, S., Santamaria, R., Theron, R., Quintales, L. and Leisch, F. (2009) biclust: Bicluster algorithms. R package version 0.7, 2.

26. Zhang, Y., Xie, J., Yang, J., Fennell, A., Zhang, C. and Ma, Q. (2016) QUBIC: a bioconductor package for qualitative biclustering analysis of gene co-expression data. Bioinformatics, btw635.

27. Eren, K., Deveci, M., Küçüktunç, O. and Çatalyürek, Ü.V. (2013) A comparative analysis of biclustering algorithms for gene expression data. Briefings in bioinformatics, 14, 279292. 
768

769

770

771

772

773

774

775

776

777

778

779

780

781

782

783

784

785

786

787

788

789

790

791

792

793

794

795

796

797

798

799

800

801

802

803

804

805

806

807

808

809

810

811

812

813

814

815

816

817

28. Chia, B.K.H. and Karuturi, R.K.M. (2010) Differential co-expression framework to quantify goodness of biclusters and compare biclustering algorithms. Algorithms for molecular biology, 5, 23.

29. Padilha, V.A. and Campello, R.J. (2017) A systematic comparative evaluation of biclustering techniques. BMC Bioinformatics, 18, 55.

30. Saelens, W., Cannoodt, R. and Saeys, Y. (2018) A comprehensive evaluation of module detection methods for gene expression data. Nature Communications, 9, 1090.

31. Lun, A.T., Bach, K. and Marioni, J.C. (2016) Pooling across cells to normalize single-cell RNA sequencing data with many zero counts. Genome Biol, 17, 75.

32. Bacher, R. and Kendziorski, C. (2016) Design and computational analysis of single-cell RNA-sequencing experiments. Genome biology, 17, 63.

33. Bengtsson, M., Stahlberg, A., Rorsman, P. and Kubista, M. (2005) Gene expression profiling in single cells from the pancreatic islets of Langerhans reveals lognormal distribution of mRNA levels. Genome Res, 15, 1388-1392.

34. Lu, C. and King, R.D. (2009) An investigation into the population abundance distribution of mRNAs, proteins, and metabolites in biological systems. Bioinformatics, 25, 2020 2027.

35. Hebenstreit, D., Fang, M., Gu, M., Charoensawan, V., van Oudenaarden, A. and Teichmann, S.A. (2011) RNA sequencing reveals two major classes of gene expression levels in metazoan cells. Mol Syst Biol, 7, 497.

36. Glaus, P., Honkela, A. and Rattray, M. (2012) Identifying differentially expressed transcripts from RNA-seq data with biological variation. Bioinformatics, 28, 1721-1728.

37. Rau, A. and Maugis-Rabusseau, C. (2017) Transformation and model choice for RNAseq co-expression analysis. Brief Bioinform.

38. Reuter, J.A., Spacek, D.V., Pai, R.K. and Snyder, M.P. (2016) Simul-seq: combined DNA and RNA sequencing for whole-genome and transcriptome profiling. Nature Methods.

39. Cohen, A.C. (1959) Simplified estimators for the normal distribution when samples are singly censored or truncated. Technometrics, 1, 217-237.

40. Stegle, O., Teichmann, S.A. and Marioni, J.C. (2015) Computational and analytical challenges in single-cell transcriptomics. Nat Rev Genet, 16, 133-145.

41. Bergmann, S., Ihmels, J. and Barkai, N. (2003) Iterative signature algorithm for the analysis of large-scale gene expression data. Phys Rev E Stat Nonlin Soft Matter Phys, 67, 031902.

42. Kanehisa, M., Furumichi, M., Tanabe, M., Sato, Y. and Morishima, K. (2017) KEGG: new perspectives on genomes, pathways, diseases and drugs. Nucleic Acids Res, 45, D353d361.

43. Gama-Castro, S., Salgado, H., Santos-Zavaleta, A., Ledezma-Tejeida, D., MunizRascado, L., Garcia-Sotelo, J.S., Alquicira-Hernandez, K., Martinez-Flores, I., Pannier, L., Castro-Mondragon, J.A. et al. (2016) RegulonDB version 9.0: high-level integration of gene regulation, coexpression, motif clustering and beyond. Nucleic Acids Res, 44, D133-143.

44. Overbeek, R., Begley, T., Butler, R.M., Choudhuri, J.V., Chuang, H.-Y., Cohoon, M., de Crécy-Lagard, V., Diaz, N., Disz, T., Edwards, R. et al. (2005) The Subsystems Approach to Genome Annotation and its Use in the Project to Annotate 1000 Genomes. Nucleic Acids Research, 33, 5691-5702.

45. Keseler, I.M., Mackie, A., Santos-Zavaleta, A., Billington, R., Bonavides-Martínez, C., Caspi, R., Fulcher, C., Gama-Castro, S., Kothari, A., Krummenacker, M. et al. (2017) The EcoCyc database: reflecting new knowledge about Escherichia coli K-12. Nucleic Acids Research, 45, D543-D550. 
818

819

820

821

822

823

824

825

826

827

828

829

830

831

832

833

834

835

836

837

838

839

840

841

842

843

844

845

846

847

848

849

850

851

852

853

854

855

856

857

858

859

860

861

862

863

864

865

866

46. Yan, L., Yang, M., Guo, H., Yang, L., Wu, J., Li, R., Liu, P., Lian, Y., Zheng, X., Yan, J. et al. (2013) Single-cell RNA-Seq profiling of human preimplantation embryos and embryonic stem cells. Nature structural \& molecular biology, 20, 1131-1139.

47. Treutlein, B., Brownfield, D.G., Wu, A.R., Neff, N.F., Mantalas, G.L., Espinoza, F.H., Desai, T.J., Krasnow, M.A. and Quake, S.R. (2014) Reconstructing lineage hierarchies of the distal lung epithelium using single-cell RNA-seq. Nature, 509, 371.

48. Dennis, G., Sherman, B.T., Hosack, D.A., Yang, J., Gao, W., Lane, H.C. and Lempicki, R.A. (2003) DAVID: database for annotation, visualization, and integrated discovery. Genome biology, 4, R60.

49. Shalek, A.K., Satija, R., Shuga, J., Trombetta, J.J., Gennert, D., Lu, D., Chen, P., Gertner, R.S., Gaublomme, J.T., Yosef, N. et al. (2014) Single-cell RNA-seq reveals dynamic paracrine control of cellular variation. Nature, 510, 363-369.

50. Ståhl, P.L., Salmén, F., Vickovic, S., Lundmark, A., Navarro, J.F., Magnusson, J., Giacomello, S., Asp, M., Westholm, J.O., Huss, M. et al. (2016) Visualization and analysis of gene expression in tissue sections by spatial transcriptomics. Science, 353, 78-82.

51. Zechel, S., Zajac, P., Lonnerberg, P., Ibanez, C.F. and Linnarsson, S. (2014) Topographical transcriptome mapping of the mouse medial ganglionic eminence by spatially resolved RNA-seq. Genome Biol, 15, 486.

52. Kiselev, V.Y., Kirschner, K., Schaub, M.T., Andrews, T., Yiu, A., Chandra, T., Natarajan, K.N., Reik, W., Barahona, M. and Green, A.R. (2017) SC3: consensus clustering of single-cell RNA-seq data. Nature methods.

53. Barrett, T., Wilhite, S.E., Ledoux, P., Evangelista, C., Kim, I.F., Tomashevsky, M., Marshall, K.A., Phillippy, K.H., Sherman, P.M. and Holko, M. (2013) NCBI GEO: archive for functional genomics data sets-update. Nucleic acids research, 41, D991-D995.

54. Barrett, T., Troup, D.B., Wilhite, S.E., Ledoux, P., Rudnev, D., Evangelista, C., Kim, I.F., Soboleva, A., Tomashevsky, M. and Edgar, R. (2007) NCBI GEO: mining tens of millions of expression profiles-database and tools update. Nucleic acids research, 35, D760D765.

55. Trapnell, C., Pachter, L. and Salzberg, S.L. (2009) TopHat: discovering splice junctions with RNA-Seq. Bioinformatics, 25, 1105-1111.

56. Trapnell, C., Williams, B.A., Pertea, G., Mortazavi, A., Kwan, G., Van Baren, M.J., Salzberg, S.L., Wold, B.J. and Pachter, L. (2010) Transcript assembly and quantification by RNA-Seq reveals unannotated transcripts and isoform switching during cell differentiation. Nature biotechnology, 28, 511-515.

57. Sha Cao, T.S., Xin Chen, Qin Ma, Chi Zhang. (2017) A probabilistic model-based biclustering method for single-cell transcriptomic data analysis. bioRxiv.

58. Tirosh, I., Izar, B., Prakadan, S.M., Wadsworth, M.H., 2nd, Treacy, D., Trombetta, J.J., Rotem, A., Rodman, C., Lian, C., Murphy, G. et al. (2016) Dissecting the multicellular ecosystem of metastatic melanoma by single-cell RNA-seq. Science, 352, 189-196.

59. Castillo-Davis, C.I. and Hartl, D.L. (2003) GeneMerge-post-genomic analysis, data mining, and hypothesis testing. Bioinformatics, 19, 891-892.

60. Monk, J., Nogales, J. and Palsson, B.O. (2014) Optimizing genome-scale network reconstructions. Nat Biotechnol, 32, 447-452.

61. Sun, X. and Nobel, A.B. (2008) On the size and recovery of submatrices of ones in a random binary matrix. Journal of Machine Learning Research, 9, 2431-2453.

62. Liberzon, A., Subramanian, A., Pinchback, R., Thorvaldsdóttir, H., Tamayo, P. and Mesirov, J.P. (2011) Molecular signatures database (MSigDB) 3.0. Bioinformatics, 27, 1739-1740. 
bioRxiv preprint doi: https://doi.org/10.1101/409961; this version posted September 7, 2018. The copyright holder for this preprint (which was not certified by peer review) is the author/funder, who has granted bioRxiv a license to display the preprint in perpetuity. It is made available under aCC-BY-NC-ND 4.0 International license.

867

868

869

870

871
63. Ashburner, M., Ball, C.A., Blake, J.A., Botstein, D., Butler, H., Cherry, J.M., Davis, A.P., Dolinski, K., Dwight, S.S. and Eppig, J.T. (2000) Gene Ontology: tool for the unification of biology. Nature genetics, 25, 25-29. 